\title{
Bose-Einstein Condensation of Cooper-Pairs in the Conventional Superconductors
}

\author{
U. Köbler \\ Research Center Jülich, Institute PGI, 52425 Jülich, Germany \\ E-mail: u.koebler@fz-juelich.de
}

Received 19 March 2021, Revised 12 May 2021, Re-revised 28 May 2021, Accepted 30 May 2021

\begin{abstract}
Available data of the temperature dependence of the superconducting heat capacity and of the thermal conductivity of the conventional superconductors are analyzed in detail. It is shown that in contrast to the exponential function predicted by the BCS theory, the temperature dependence of the superconducting heat capacity consists of a sequence of a few analytically different universal power functions of absolute temperature. The changes from one to the next power function are typical examples of crossover events. The crossover occurring at the lowest temperature, commonly below about $\sim 1 \mathrm{~K}$, is identified as transition from Maxwell-Boltzmann to Bose-Einstein (BE) statistics of the Cooper-pairs. Because of the low mass of the Cooper pairs of $2 \mathrm{~m}_{\mathrm{e}}$ (with $\mathrm{m}_{\mathrm{e}}$ as the mass of the electron) and their high density, the $\mathrm{BE}$-condensation temperature, $\mathrm{T}_{\mathrm{BE}}$, of the Cooper-pairs is about five orders of magnitude higher than for the dilute alkali atom condensates. The condensation temperature $\mathrm{T}_{\mathrm{BE}}$ turns out to be proportional to the superconducting transition temperature $T_{\mathrm{SC}}$. Since $T_{\mathrm{BE}}$ is proportional to $\sim \mathrm{n}^{2 / 3}$, with $\mathrm{n}$ as the density of the Cooper pairs at $T_{\mathrm{BE}}$, it is possible to obtain the density of the Cooper pairs at low temperatures. Assuming that for the type I superconductors the Cooper pairs form a dense gas of bosons with virtually no space between them, the diameter of the Cooper-pair orbital, calculated from $\mathrm{n}^{2 / 3}$, turns out to agree quantitatively with the experimental value of the London penetration depth. As a conclusion, due to the large orbital diamagnetism of the Cooper-pairs, only one layer of Cooper-pairs, next to the inner surface of the sample, is sufficient to shield an applied external magnetic field completely.
\end{abstract}

\section{Keywords: Bose-Einstein condensation; conventional superconductors; heat capacity; thermal conductivity}

\section{Introduction}

The postulation of Cooper-pairs marks a breakthrough in our understanding of the phenomenon of superconductivity, whatever the detailed mechanism of the attractive interaction between the two Cooper-pair electrons in the various types of superconductors is [1-9]. As was evidenced by the BCStheory, the superconducting excitation gap is a measure of the coupling strength between the two electrons of the Cooper-pair and therefore correlates with the superconducting transition temperature $\mathrm{T}_{\mathrm{SC}}$ [1]. Emergence of Cooper-pairs at $\mathrm{T}_{\mathrm{SC}}$ is associated with the rise of a large diamagnetism due to the large cross section of the Cooperpair orbital. As a consequence, the two Cooper-pair electrons are in a spin-compensated singlet state and circulate on a closed loop. Approaching $\mathrm{T}_{\mathrm{SC}}$ from the low-temperature side, the interaction between the two Cooper-pair electrons decreases, in parallel with the excitation gap, and the diameter of the Cooper pair orbital increases and, eventually, diverges at $\mathrm{T}_{\mathrm{SC}}$. The increasing orbital diameter correlates with the behavior of the London penetration depth for an applied magnetic field (see Figure 10 below) [2,3]. With decreasing temperature, the interaction between the two Cooper-pair electrons and the excitation gap increase and the diameter of the Cooper-pair orbital shrinks [3]. We have every reason to assume that at the same time the density of the Cooper pairs, n, increases correspondingly. In other words, the Cooper-pairs form dense-packed gas of particles. At least for the type I superconductors there seems to be no free space between the Cooper pairs that would allow magnetic field lines (vortices) to penetrate the superconductor.

A completely new chapter of solid-state physics began with the development of the Renormalization Group (RG) theory at about 1974 [10]. Originally developed to explain the critical behavior of spin systems in the vicinity of the magnetic ordering temperature, the principles of the $R G$ theory turned out to be of quite general importance for the dynamics of all energy degrees of freedom of the solids (magnetic, elastic, electronic...). One important issue of the RG theory was that the dynamics in the vicinity of a critical temperature is the dynamics of a boson field, exclusively. The atomistic near neighbor interactions are completely excluded from the critical dynamics. The bosons are massless particles that propagate ballistic, i.e. independent of the lattice structure and of the chemical composition of the solid. This is the origin of the material-independent universality of the critical dynamics. RG theory was, however not able to specify the bosons that are responsible (relevant) for the critical magnetic dynamics. As could be shown recently, these bosons are essentially magnetic dipole radiation, generated basically through stimulated emission by the precessing spins [11]. We have called these bosons Goldstone bosons [12]. The bosons of the elastic degree of freedom are the well-known sound waves. We will call them Debye bosons [13]. The bosons of the metallic degree of freedom are completely unexplored. One reason for the generally poor information about the bosons is that mass-less bosons cannot be investigated using neutron scattering. 
In our context it is important to note that $\mathrm{T}=0$ is a critical temperature, completely equivalent to a finite ordering temperature. The critical power functions at $\mathrm{T}=0$ are power functions of absolute temperature. This is well-known for the heat capacity of the Debye boson field $\left(\sim \mathrm{T}^{3}\right)$ and for the heat capacity of the bosons of the continuous metallic solid $(\sim \mathrm{T})$. We will call the latter bosons CMS-bosons [14]. Note that the two mentioned power functions hold rather precisely over a finite temperature range, up to a crossover temperature at which thermal energy changes from the boson field into the corresponding atomistic excitation system, i.e. to the acoustic phonons and to the electron-band states, respectively [14]. At this crossover, an analytical change in the temperature dependence the heat capacity occurs and the two power functions of absolute temperature cease [15]. For higher temperatures, the heat capacity is material specific. This crossover, commonly occurs at a temperature of $10 \ldots 30$ $\mathrm{K}$ [14]. For the unusual superconductor $\mathrm{MgB}_{2}$ with $\mathrm{T}_{\mathrm{SC}}$ as high as $\mathrm{T}_{\mathrm{SC}}=39 \mathrm{~K}$, this crossover is at a surprisingly high temperature of larger than $80 \mathrm{~K}$ [16].

The atomistic excitations (phonons, electron-band states) and the corresponding bosons (Debye-bosons, CMS-bosons) differ fundamentally by their translational symmetry that is discrete-periodic for the atomistic excitations but continuous for the bosons. Note that for the bosons there are no atoms, the zone-boundary is of no importance [13]. In principle, the two symmetries exclude each other. This means, the observed dynamics is either the dynamics of the bosons or the dynamics of the atomistic excitations. According to the symmetry selection principle of relevance, borne out by the Renormalization Group theory [10], within the critical temperature range at $\mathrm{T}=0$ or at a finite ordering temperature, the bosons are the relevant excitations. In other words, in the critical range, all thermal energy is in the boson field, and the dispersion relations of the atomistic excitations are thermally not populated. However, in order that the thermal energy is either in one or the other excitation system, and the dynamic symmetry therefore is clearly defined as continuous or discrete, it is necessary that the thermal energy can change between the two excitation systems. This requires a finite interaction between the two systems.

The superconducting transition temperatures, $\mathrm{T}_{\mathrm{SC}}$, of the elements (Al, Ga, In, Nb, Pb, Sn, Ta, V...) are all sufficiently low to fall in the critical range near $\mathrm{T}=0$ [17]. As a consequence, the dynamics above and below $\mathrm{T}_{\mathrm{SC}}$ is exclusively due to bosons, and requires field theories, instead of atomistic concepts, for an adequate description. Universality of the linear-in- $\mathrm{T}$ function and of the $\mathrm{T}^{3}$ function means, that all microscopic and material specific details such as the lattice structure and the inter-atomic interactions are not important (relevant) for the temperature dependence of the thermodynamic observables.

From the linear superposition of the heat capacities of the Debye-boson field $\left(\sim \mathrm{T}^{3}\right)$ and of the CMS-boson field $(\sim T)$ for $\mathrm{T} \geq \mathrm{T}_{\mathrm{SC}}$ [18] it follows that the two boson types do virtually not interact. For a finite interaction, the boson type with the lower dispersion energy would take over all thermal energy and a single power function of absolute temperature with the universal exponent characteristic of the low-energy system would be observed. A possible finite contribution of the non-relevant system can increase the pre-factor of the universal power function of the relevant system [15,21].

While the critical exponents are universal, i.e. material independent, the pre-factors of the universal power functions of temperature are material-specific. This is indicative of a finite coupling of the bosons to the atomistic back-ground. In other words, the bosons cannot be treated as free particles. In fact, the stiffness constant of the linear dispersion of the Debye bosons and therefore their absolute velocity is material dependent and, commonly, agrees with the initially linear dispersion of the acoustic phonons [19]. Since the dispersion relation of the CMS-bosons is not known, the heat capacity provides the only experimental access to the CMSbosons. Note that the dispersion relations of mass-less bosons cannot be investigated using neutron scattering.

Characteristic of the superconducting transition is that neither in the heat capacity (see Figures 1-4 below) nor in the critical field [20] critical power functions of the argument $\left|\mathrm{T}_{\mathrm{SC}}-\mathrm{T}\right|$ occur, as they are typical for a phase transition into a long-range and coherently ordered state [21]. Instead, only power functions of absolute temperature are observed above and below $\mathrm{T}_{\mathrm{SC}}$. This shows that $\mathrm{T}=0$ is the only critical point, in the sense of the RG-theory. As is well known, upon approaching $\mathrm{T}_{\mathrm{SC}}$ from the high-temperature side, the $\mathrm{T}^{3}$ function and the linear-in-T function hold precisely until the discontinuity at $\mathrm{T}_{\mathrm{SC}}[15,18]$.

Viewing the superconducting transition as a classical mean-field transition it follows that there is no coherent longrange order in the superconducting state. Instead, there is short-range order only. Note that short-range order is typical for the critical range, either above $\mathrm{T}=0$ or above a finite ordering temperature. In fact, we can consider the Cooper pairs $[3,4]$ as the short-range ordered objects. This behavior is in contrast to the boson executed magnetic ordering transitions, at which the typical critical power functions of the argument $\left|\mathrm{T}_{\mathrm{c}}-\mathrm{T}\right|$ are observed [21,22]. As we have mentioned, universality of the critical magnetic dynamics above and below $\mathrm{T}_{\mathrm{c}}$ implies that the atomistic near-neighbor interactions are excluded from the critical dynamics. As is well-known, for ferromagnets and for antiferromagnets of the same symmetry class, the critical exponents are identical. As a consequence, the ordering transition is a phenomenon of the boson field. In fact, at the magnetic ordering transition, the Goldstone-boson field orders [22]. Due to a finite interaction of the spins with the boson field, the spins order at the same time. At the magnetic ordering transition, the density of the Goldstone-bosons has reached the threshold value for stimulated emission to become the decisive process and the Goldstone-boson field orders perfectly onedimensional along each magnetic domain axis. Typical for the ordered state of the Goldstone-boson field are domains, which have not been identified in the superconductors [17]. The boson field in each magnetic domain resembles the beam of a LASER. A three-dimensional global boson field results by a coupling of the one-dimensional boson fields of the domains along $\mathrm{x}-, \mathrm{y}-$ and $\mathrm{z}$-axis. In other words, the dimensionality of the global boson field corresponds to the number of inequivalent domain orientations. In a twodimensional magnet there are domains along $\mathrm{x}$ - and $\mathrm{y}$-axis only. The coherent and long-range ordered spin system results from a strong interaction of the spins with the coherent and one-dimensional boson field [22]. In fact, stimulated emission seems to be an important mechanism for the phenomenon of broken symmetry. Note that the microscopic interactions between atoms or spins are well able to drive an ordering transition but this transition is not associated with the formation of domains and therefore is not into a state with a coherent long-range order [12,22]. In reality, all magnetic ordering transitions of the magnets with 
a three-dimensional spin are boson driven and exhibit universal critical dynamics.

The CMS-bosons interact very little with all other degrees of freedom, and their mean free path is correspondingly large. This can be concluded from the fact that the lowtemperature thermal conductivity of all normal conducting metals is exclusively due to the CMS-bosons and starts, at low-temperatures, with the same linear-in-T dependence as the heat capacity of the CMS-bosons $[14,15]$. This shows that the mean free path of the CMS-boson is larger than the linear dimension of the samples used in the thermal conductivity measurements. The Debye bosons do not contribute at all to the thermal conductivity of the normal conducting metals, in spite of their finite heat capacity (in the temperature range of the $\mathrm{T}^{3}$ function).

On the other hand, thermal conductivity of the insulators is exclusively due to the Debye bosons [14]. In contrast to the CMS-bosons, the Debye bosons are strongly coupled to the (acoustic) phonons [19]. Only for a few known insulators with a high thermal conductivity, thermal conductivity starts with the $\mathrm{T}^{3}$ function of the heat capacity of the Debye-boson field $[14,19]$. Phonons do not contribute at all to the thermal conductivity of the insulators and of the metals as well [14]. In the harmonic approximation, phonons are standing waves.

A high thermal conductivity of the metals and of the insulators requires that the CMS-bosons and the Debyebosons are relevant. This means, they must have a heat capacity. This holds for the low-temperature range of the linear-in- $T$ function and of the $\mathrm{T}^{3}$ function in the heat capacity only [15].

At the superconducting transition temperature $\mathrm{T}_{\mathrm{SC}}$, the interactions between all involved sub-systems change. In particular, the Debye-bosons and the CMS-bosons seem to interact significantly. As a consequence, the heat capacities of the two boson fields do no longer superimpose for $\mathrm{T}<\mathrm{T}_{\mathrm{SC}}$. We can assume that thermal conductivity below $\mathrm{T}_{\mathrm{SC}}$ is still dominated by the CMS-bosons but due to the interaction with the Debye-bosons, the mean free path of the CMSbosons becomes shorter and thermal conductivity is lower in the superconducting state compared to the normal conducting state [23]. Another sudden change occurring at the superconducting transition concerns the electron-electron interaction which is repulsive above $\mathrm{T}_{\mathrm{SC}}$ but attractive below $\mathrm{T}_{\mathrm{SC}}$ such that Cooper-pairs are formed [1,3]. It is tempting to assume that one possible coupling mechanism between the two electrons of the Cooper-pair could be essentially by exchange of Goldstone-bosons (magnetic dipole radiation).

As an empirical fact, the temperature dependence of the superconducting heat capacity can well be described by a sequence of universal power functions of absolute temperature each of which has a rational exponent and holds over a finite temperature range [15]. This is typical for boson dynamics. The changes from one to the next power function of temperature are textbook examples of crossover events $[19,22]$. The various observed rational exponents seem to depend in a complicated way on the densities of states and on the interactions between the involved sub-systems, and therefore are difficult to understand [19,22]. Although quantities such as the density of states can be assumed to change monotonically as a function of energy (temperature), the boson-defined dynamics reacts in the discrete manner of crossover events. At these crossovers the exponent in the power functions of absolute temperature changes but remains always a rational number. As examples of this behavior, Figures 1-4 show for a selection of superconducting elements the temperature dependence of the superconducting heat capacity in a semi-logarithmic representation as a function of $\mathrm{T}_{\mathrm{SC}} / \mathrm{T}$. Since in this plot the experimental data fall on curved lines throughout, the linear dependence expected for the exponential function according to the BCS theory, does not hold. This is no surprise since, as for $\mathrm{T} \geq \mathrm{T}_{\mathrm{SC}}$, the superconducting heat capacity is the heat capacity of boson fields for $\mathrm{T} \leq \mathrm{T}_{\mathrm{SC}}$ as well. Bosons are, however, not included in the BCS theory. Note that bosons always have gap-less excitation spectra, given by a power function of wave-vector with a rational exponent [12,22]. As a consequence, the temperature dependence of the heat capacity of the boson fields is given by a power function of absolute temperature, also with a rational exponent. Although the energy of the superconducting gap is indicative of the coupling strength between the two Cooper-pair electrons, the gap is not relevant for the dynamics. This type of behavior we know from the ordered magnets. For instance, in the axial antiferromagnet $\mathrm{MnF}_{2}$ a magnon excitation gap of $\sim 1.1 \mathrm{meV}(\sim 13 \mathrm{~K})$ occurs [15]. Nevertheless, thermal decrease of the spontaneous magnetization is given by the heat capacity of the Goldstonebosons, and does not follow an exponential function but a $\mathrm{T}^{5 / 2}$ power function over a large temperature range $[12,15,22]$. It is therefore evident that, at the lowest temperatures, the power function of the bosons results in much larger heat capacity values than the exponential function of the BCS-theory. This disagreement with the BCS-theory has already been noticed for lead in 1965 [24]. Lead is a mechanically soft metal with a very low Debye temperature of $\Theta_{\mathrm{D}} \sim 100 \mathrm{~K}[17,25]$. As a consequence, the heat capacity of the Debye boson field is much larger than the heat capacity of the CMS-boson field such that the exponent of three of the Debye boson field dominates the superconducting heat capacity at the lowest temperatures. Moreover, for higher temperatures the dynamics is bosonic as well and the heat capacity exhibits a crossover to a power function of absolute temperature with another exponent $[15,19,22]$. Note that the non-asymptotic power functions include an absolute constant. Since the Cooper-pairs are atomistic objects they receive their dynamics -as the spins in the ordered magnets- from the relevant boson fields.

Cooper pairs are massive bosons with an integer spin of $\mathrm{S}=0$ [4]. Treating the Cooper pairs as an ideal gas, an estimate for the crossover temperature from MaxwellBoltzmann to Bose-Einstein statistics $\left(\mathrm{T}_{\mathrm{BE}}\right)$ can be obtained from the condition that the de Broglie wavelength of the Cooper-pairs, $\lambda_{\mathrm{dB}}$, must be equal or larger than the mean distance between the Cooper pairs. Expressing the mean distance between the Cooper-pairs by their density, n, the condition for BE-statistics reads:

$$
\lambda_{\mathrm{dB}}>\mathrm{n}^{-1 / 3}
$$

The de Broglie wave-length is equal to $\lambda_{\mathrm{dB}}=\mathrm{h} / \mathrm{p}=\mathrm{h} /(2 \mathrm{E} \cdot \mathrm{m})^{1 / 2}$ with an average thermal energy that can be assumed to be proportional to $\mathrm{E} \sim \mathrm{k}_{\mathrm{B}} \mathrm{T}$. The proportionality factor depends on the details of the system. From quantitative calculations [26] for the uniform Bose gas, confined to a three-dimensional box, the Bose-Einstein temperature comes out as:

$$
\mathrm{T}_{\mathrm{BE}}<0.084 \cdot \mathrm{h}^{2} \cdot \mathrm{n}^{2 / 3} / \mathrm{m} \cdot \mathrm{k}_{\mathrm{B}}
$$


with h as Planck's constant and $\mathrm{k}_{\mathrm{B}}$ as Boltzmann constant. This equation is proven as quantitatively correct for a dilute gas of spin-polarized atomic hydrogen with a density of $\mathrm{n}=1.8 \cdot 10^{14} \mathrm{~cm}^{-3}$ particles [27]. For this particle density the $\mathrm{BE}$ temperature calculated according to Eq. (2) is $\mathrm{T}_{\mathrm{BE}}=51$ $\mu \mathrm{K}$, inserting as mass the mass of the proton. This value agrees perfectly with the observed $\mathrm{BE}$ temperature of $\mathrm{T}_{\mathrm{BE}}=50 \mu \mathrm{K}$ [27].

Note that in contrast to the dilute alkali atom condensates [2], the Cooper pair density, $n$, is temperature dependent. Moreover, for the Cooper pairs we have to insert into Eq. (2) as mass of the bosons $m=2 m_{e}$ with $m_{e}$ as the mass of the electron. Since the mass of the proton is about a factor of 2000 larger than the mass of the electron, and a typical atomic mass for the alkali atoms is $\sim 50$, the denominator in Eq. (2) is smaller for the Cooper-pairs by about a factor of $10^{5}$ compared to the alkali atoms. Additionally, we can assume that the Cooper-pairs form a dense gas with a particle density at $\mathrm{T}_{\mathrm{BE}}$ of higher by a factor of 10 to 20 compared to the particle density of the dilute alkali gases [26]. The BEtemperature of the Cooper pairs therefore is larger by about six orders of magnitude compared to the dilute alkali atom gases, and therefore falls in an experimentally much easier to realize temperature range. For a typical BE-temperature of $\mathrm{T}_{\mathrm{BE}} \sim 0.5 \mathrm{~K}$ observed in the heat capacity of the superconducting elements a density of the Cooper-pairs, at $\mathrm{T}_{\mathrm{BE}}$, of $\mathrm{n}=6.4 \cdot 10^{15} \mathrm{~cm}^{-3}$ follows according to Eq. (2). The mean distance between the Cooper-pairs therefore is $\lambda=n^{-1 / 3}$ $=54 \mathrm{~nm}$. This value is of the order of the experimentally observed London penetration depths (see Figure 10 below) [17]. Assuming that the Cooper-pairs form a dense gas of bosons, the linear dimension of the Cooper pairs agrees with their distance [4]. In other words, only one layer of Cooperpairs at the inner surface of the superconductor appears to be sufficient to shield an applied magnetic field completely. This is a consequence of the large orbital diamagnetism of the Cooper-pairs.

Although it is well established that the phenomenon of superconductivity bases on the emergence of Cooper-pairs at the superconducting transition temperature, there is still a strong need to obtain more detailed experimental information on their nature. In the present study it will be shown that quantitative analyses of the BE condensation temperatures of the Cooper pairs allow one to obtain rather precise data of their size and density. Surprisingly, in spite of their two-fold charge, the BE condensation of the Cooperpairs can be described using the same concepts as were developed for the condensation of the neutral alkali metal atoms.

\section{Analysis of experimental data}

In order to illustrate the experimental characteristics of boson dynamics, and therefore the discrepancies to the atomistic BCS theory, Figures 1 to 4 display published heat capacity data of the superconducting range of vanadium [28], gallium [29], aluminum [30] and indium [31, 32], respectively. In these Figures the natural logarithm of the asmeasured superconducting heat capacity is plotted as a function of $\mathrm{T}_{\mathrm{SC}} / \mathrm{T}$ with $\mathrm{T}_{\mathrm{SC}}$ as superconducting transition temperature. As can be seen in Figures 1-4, for all temperatures the superconducting heat capacity data are on curved lines that can excellently be described by a sequence of power functions of absolute temperature with different rational exponents. If the data would follow the exponential function predicted by the BCS-theory, a straight line should result in these plots. We should recall that it is not possible to decompose the experimental heat capacity into an electronic contribution due to the CMS-bosons and an elastic contribution due to the Debye-bosons, simply because the two degrees of freedom interact significantly in the superconducting state. Note that all fit functions have to include an absolute constant [13,22]. An absolute constant indicates that the observed power function is not the asymptotic behavior for $\mathrm{T} \rightarrow 0$ and that one or more further crossover events can be expected to follow at lower temperatures. Only the asymptotic power function does not include a significant absolute constant. In the temperature window shown in Figures 1-4, at least one crossover event between two power functions of absolute temperature with different exponents is identified. At the temperatures of the conventional superconductors, phonons are not relevant, and completely negligible. Within the error limits, the adopted rational exponents are consistent with the given fit values.

The crossover events in Figures 1-4 are rather smooth functional changes and require a very careful look on the experimental data for their identification. In particular, the precise identification of the validity limits of each power function is crucial for a reliable fit-result for the exponent. Since we are rather sure that all exponents are rational numbers, one can be confident in the fitting procedure if the obtained exponent comes out as a rational number within the experimental error limits.

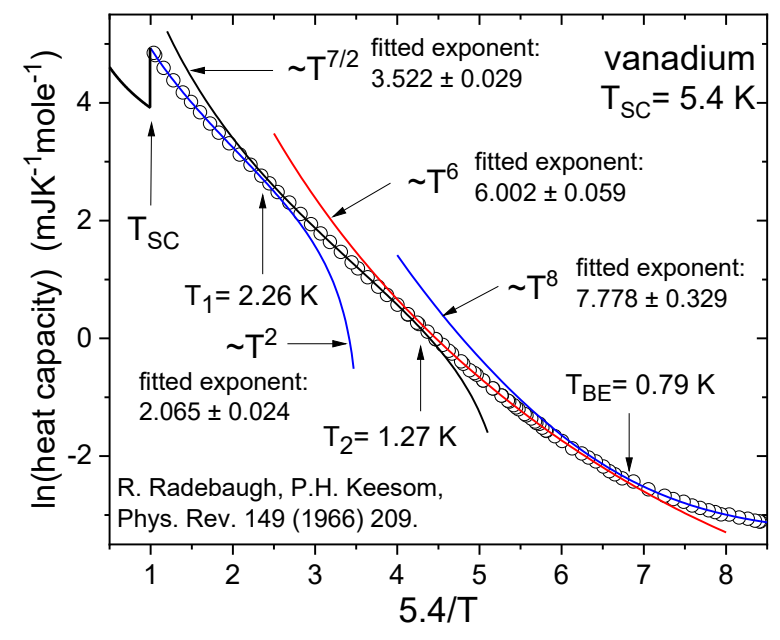

Figure 1. Natural logarithm of the as-measured heat capacity of the superconducting state of vanadium as a function of $T_{S C} / T$ with $T_{S C}=5.4 \mathrm{~K}$ as superconducting transition temperature [28]. Four sections with power functions of $T^{2}, T^{7 / 2}, T^{6}$ and $T^{8}$ can be distinguished. The crossover at the lowest temperature is identified as the BoseEinstein crossover temperature $T_{B E}$ (see Figure 9).

In the case of vanadium (Figure 1), the four power functions $\mathrm{T}^{2}, \mathrm{~T}^{7 / 2}, \mathrm{~T}^{6}$ and $\mathrm{T}^{8}$ can be identified in the temperature dependence of the superconducting heat capacity. This implies three crossover events. As we have already mentioned, the condition for a crossover event to occur as well as the exponents involved are difficult to understand.

The exponent of three in the asymptotic power function of the mechanically soft metal indium (Figure 4) can be assumed to be dominated, as for lead [24], by the enormous large heat capacity of the Debye-bosons, according to a Debye-temperature of $\Theta_{\mathrm{D}} \sim 110 \mathrm{~K}$ only $[17,25]$. As we have 
mentioned, finite but non-relevant heat capacity contributions can increase the pre-factor of the $\mathrm{T}^{3}$ function [21].

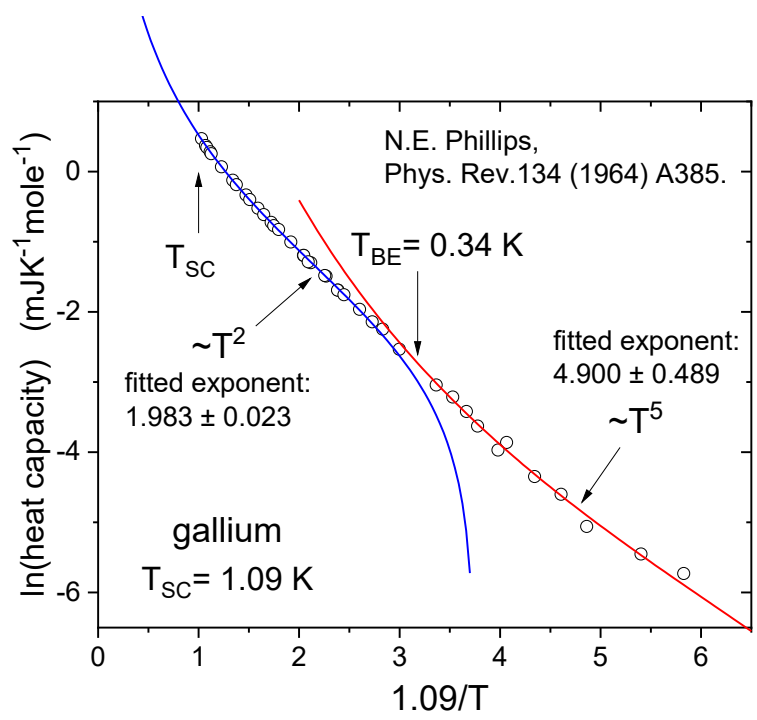

Figure 2. Natural logarithm of the as-measured heat capacity of the superconducting state of gallium plotted as a function of $T_{S C} / T$ with $T_{S C}=1.09 \mathrm{~K}$ as superconducting transition temperature [29]. The crossover from $T^{2}$ function to $T^{5}$ function is identified as Bose-Einstein temperature $T_{B E}$ (see Figure 9).

There is one remarkable difference between Figure 4 and the Figures 1-3: the two power functions in Figure 4 cross (see Figure 7) while the power functions in the Figures 1 to 3 avoid each other and approach tangentially only. Crossing means that there is either no interaction between the excitations giving rise to the two heat capacity functions (which can be excluded) or both excitations have the same symmetry. Note that power functions pertinent to excitations with different symmetries cannot cross if there is a finite interaction between them. If, by symmetry reasons, the power functions are not allowed to cross, we must assume that the observed exponents could be affected by the noncrossing condition [21]. This makes the interpretation of the observed exponents additionally difficult.

As an empirical fact, the Bose-Einstein transitions of the superconducting elements give rise to a similarly weak anomaly in the heat capacity as the other crossover events seen in Figures 1- 4. A clear discontinuity is not observed in the heat capacity at $\mathrm{T}_{\mathrm{BE}}[8,9,26]$. This behavior conforms to the model of a Bose gas of non-interacting particles, confined to a three-dimensional box [26]. For this model, the heat capacity is continuous at $\mathrm{T}_{\mathrm{BE}}$ but the first derivative with respect to temperature is discontinuous. The problem therefore is to identify the BE transition among the other observed crossover events. Commonly, however, the BoseEinstein crossover is the crossover at the lowest temperature. The consistency of this criterion will be rationalized below (see Figures 9 and 10).

A considerable problem with boson dynamics is that the bosons get scattered at all types of lattice imperfections. This proves some coupling of the bosons to the atomistic background. Scattering provides damping to the bosons and decreases their velocity and therefore changes their dispersion relation. Strain can additionally affect the dynamics of the boson field. Fortunately, boson dynamics holds under all conditions but the observed universal exponents and the crossover temperatures between the various power functions of temperature can be sample dependent. Note that this is in contrast to the atomistic dynamics of the phonons that is determined by the local interactions between the atoms and therefore is less sensitive to strain or to the mosaic structure of the sample. Strain is a phenomenon on the larger length scale of the bosons. In the elastic case, sample-dependent effects are well-known from the sound velocities, and therefore from the elastic constants. Due to this non-intrinsic effect, the elastic constants get additionally decreased as a function of increasing temperature and therefore are not perfectly reproducible [34].

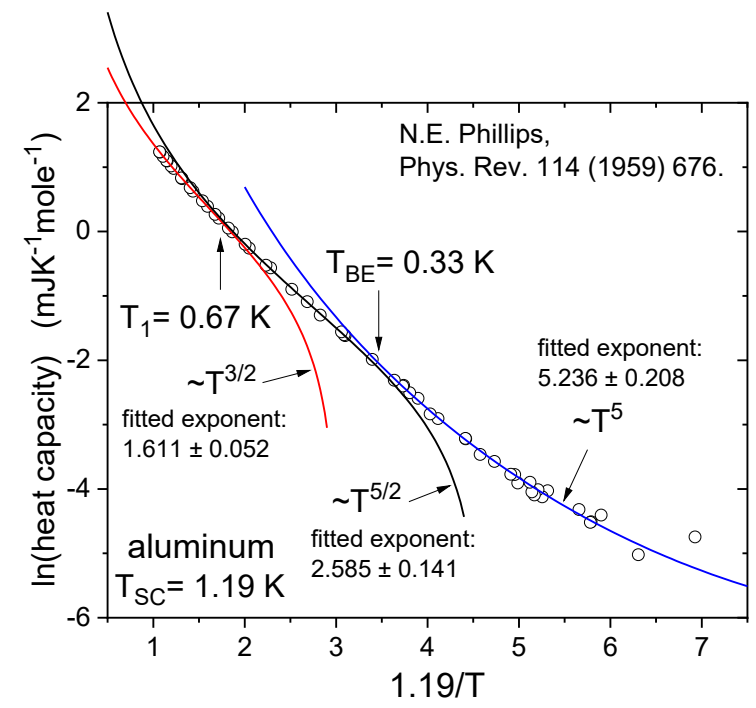

Figure 3. Natural logarithm of the as-measured heat capacity of the superconducting state of aluminum plotted as a function of $T_{S C} / T$ with $T_{S C}=1.19 \mathrm{~K}$ as superconducting transition temperature [30]. Three sections with power functions of $T^{3 / 2}, T^{5 / 2}$ and $T^{5}$ can be distinguished. The crossover from $T^{5 / 2}$ to $T^{5}$ function is identified as BoseEinstein temperature $T_{B E}=0.33 \mathrm{~K}$ (see Figures 8 and 9).

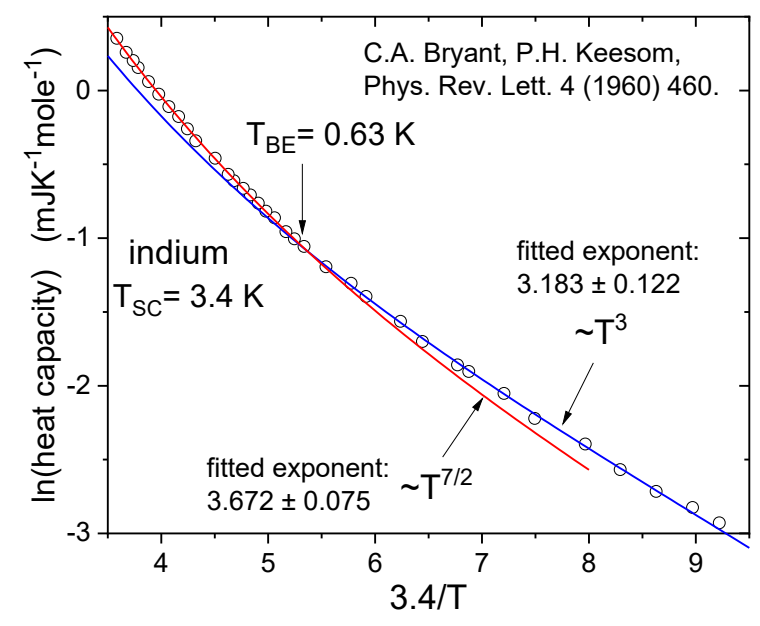

Figure 4. Low-temperature heat capacity data of the superconducting state of indium, plotted in the manner as for the Figures above [31]. The crossover from $T^{7 / 2}$ to $T^{3}$ function at $\sim 0.63 \mathrm{~K}$ is identified as Bose-Einstein crossover. A further crossover to $T^{3}$ function occurring above about $\sim 1$ $K$ is outside the temperature window on the left-hand side of this plot (see Figure 7) [31,32]. 


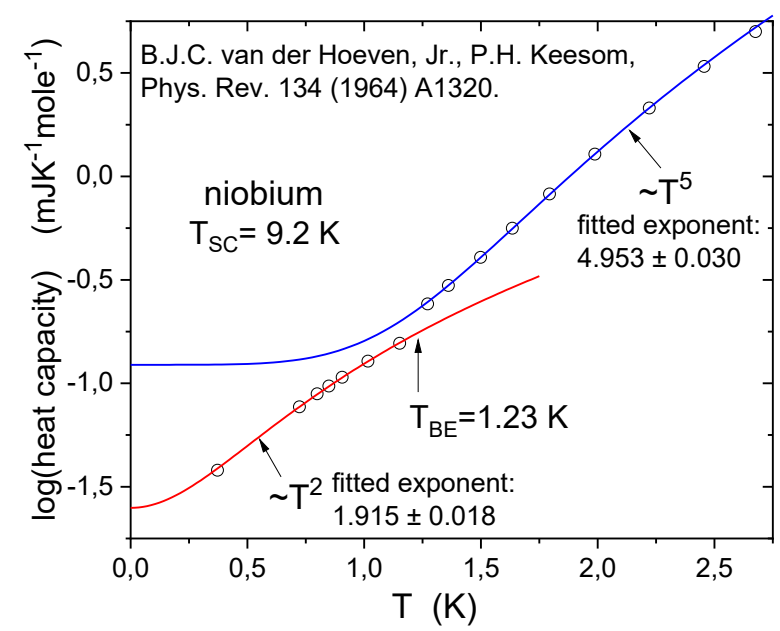

Figure 5. Logarithm to the basis of 10 of the heat-capacity of niobium in the temperature region of the BE crossover at $T_{B E}=1.23 \mathrm{~K}$ [35]. The two power functions of temperature avoid each other (see Figure 8).

In order to visualize the anomaly at the Bose-Einstein crossover temperature with a better resolution than in the preceding Figures, we now show enlarged views of the temperature regions just in the vicinity of the $\mathrm{BE}$ crossover. For niobium with the largest superconducting transition temperature of $\mathrm{T}_{\mathrm{SC}}=9.2 \mathrm{~K}$ the $\mathrm{BE}$ crossover temperature is as high as $\mathrm{T}_{\mathrm{BE}}=1.23 \mathrm{~K}$ (Figure 5) [35]. It becomes evident from Figure 5 that the extremely weak anomaly at $\mathrm{T}_{\mathrm{BE}}$ can easily be overlooked. Only with the help of the fitted power functions the crossover appears clearly.

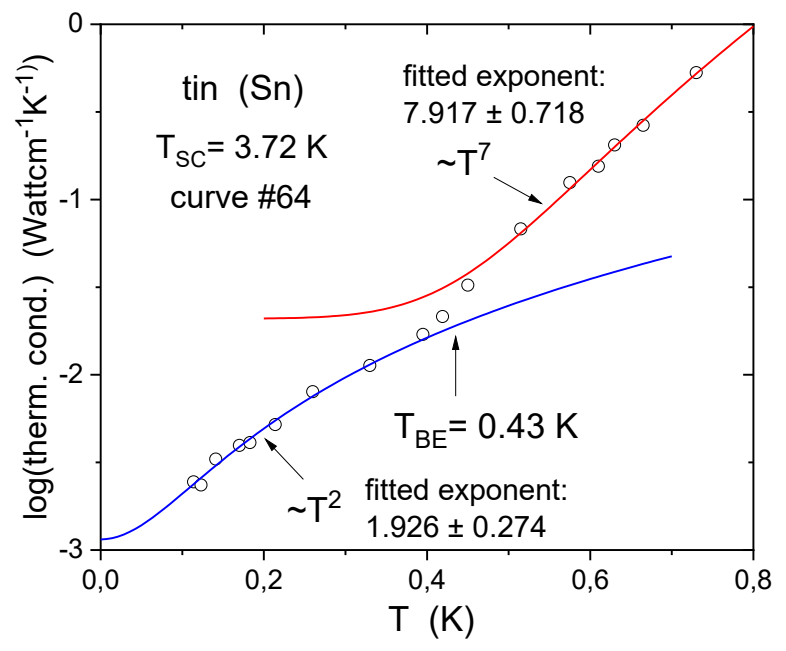

Figure 6. Logarithm to the basis of 10 of the thermal conductivity of tin in the temperature range of the $B E$ crossover at $T_{B E}=0.43 \mathrm{~K}$ [33]. The two power functions avoid each other.

For many materials, the BE crossover appears as a more pronounced anomaly in the thermal conductivity [33] rather than in the heat capacity. Only for a mean free path of the bosons of larger than the linear dimension of the sample, thermal conductivity starts with the temperature dependence the heat capacity. This, however, is scarcely the case for the low temperatures of the $\mathrm{BE}$ transition where thermal conductivity is low [23]. Figure 6 shows the logarithm to the basis of ten of the thermal conductivity of tin ( $\mathrm{Sn})$ as a function of temperature [33]. In this representation the BE crossover appears as a very similar and weak anomaly as in the heat capacity in Figure 5.

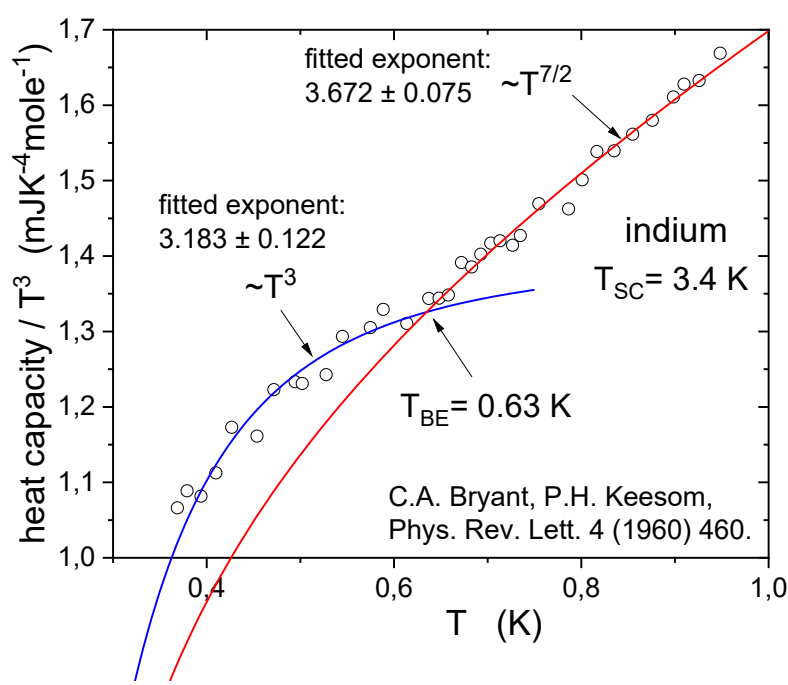

Figure 7. Superconducting heat capacity of indium divided by $T^{3}$ in the vicinity of the BE crossover temperature as a function of absolute temperature (compare Fig. 4) [31]. The two power functions cross.

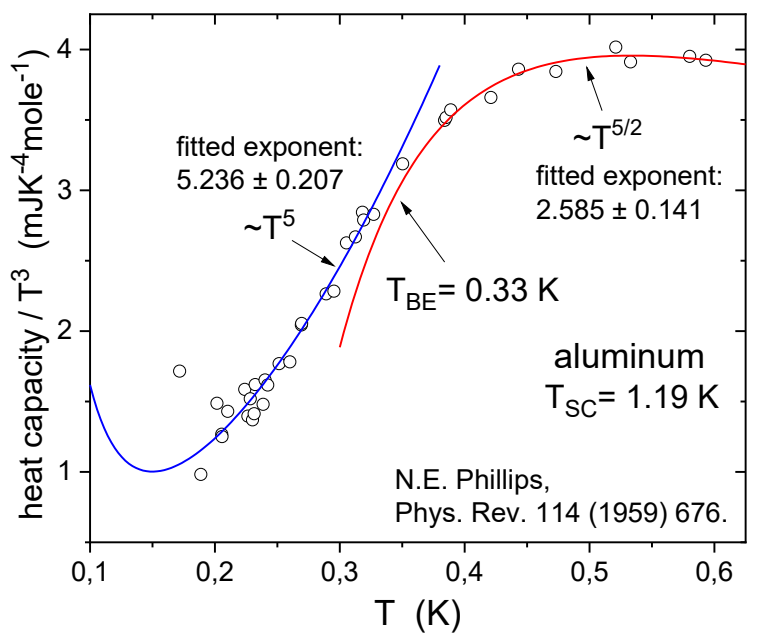

Figure 8. Superconducting heat capacity divided by $T^{3}$ of the temperature range of the $B E$ crossover at $T_{B E}=0.33 \mathrm{~K}$ of aluminum (compare Fig. 3) [30]. The two power functions avoid each other.

Another method to increase the weight of the extremely low heat capacity data at the lowest temperatures is to divide the heat capacity by the absolute temperature raised to an appropriate power. In Figure 7 the low-temperature heat capacity data in the vicinity of the BE crossover of indium have been divided by $\mathrm{T}^{3}$ and are plotted as a function of absolute temperature. As can be seen, the two power functions above and below $\mathrm{T}_{\mathrm{BE}}$ cross (compare Figure 4). This is different for the other superconductors (Figures. 5, 6, 8) for which the two power functions above and below $T_{B E}$ avoid each other.

\section{Results}

Int. Centre for Applied Thermodynamics (ICAT) 
As assumed by the BCS-theory, we use the empirical fact that the superconducting properties of the elements are all very similar, except for their transition temperatures, $\mathrm{T}_{\mathrm{SC}}$. The fitted Bose-Einstein crossover temperatures, $\mathrm{T}_{\mathrm{BE}}$, turn out to be, to a good approximation, proportional to the superconducting transition temperature, $\mathrm{T}_{\mathrm{SC}}$ (Figure 9). Qualitatively, this can be understood since when $\mathrm{T}_{\mathrm{SC}}$ is high, the Cooper-pairs are tightly bound and therefore are small objects. Since the size of the Cooper-pairs decreases with decreasing temperature, we have to assume that the number of Cooper-pairs increases in such a way with decreasing temperature that the Cooper-pairs always form a dense gas. In other words, their density, n, increases with decreasing temperature, and eventually reaches the critical density necessary for the BE-condensation to occur. As consequence, for superconductors with a high $\mathrm{T}_{\mathrm{SC}}$, the critical density for BE-condensation is reached at correspondingly high temperature.

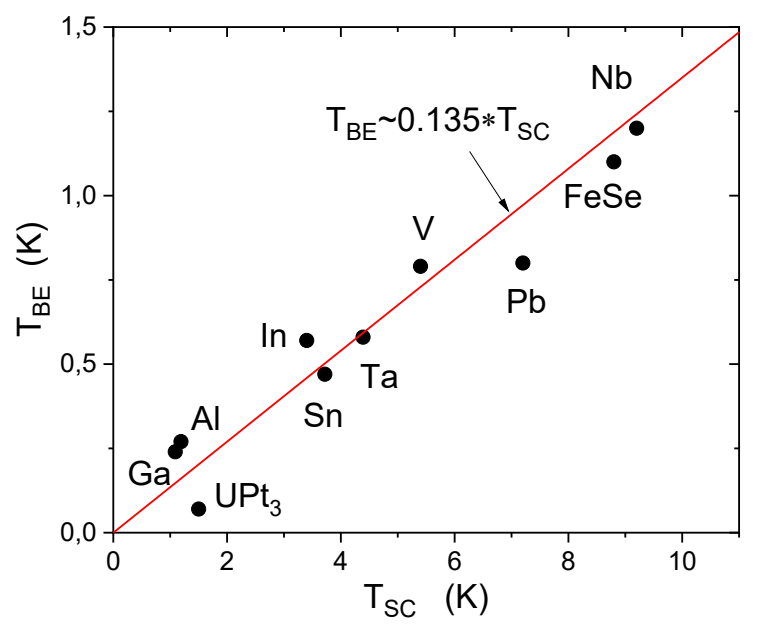

Figure 9. Bose-Einstein crossover temperature, $T_{B E}$, obtained from analyses of heat capacity and thermal conductivity data of the here investigated superconductors as a function of the superconducting transition temperature, $T_{S C}$. These data represent the average over the $T_{B E}$ temperatures, identified in the heat capacity and in the thermal conductivity [33].

According to Eq. (2) the Bose-Einstein temperature scales with the Cooper-pair density, $n$, as $\mathrm{T}_{\mathrm{BE}} \sim \mathrm{n}^{2 / 3}$. In other words, from $\mathrm{T}_{\mathrm{BE}} \sim \mathrm{T}_{\mathrm{SC}}$ (Figure 9) it follows that $\mathrm{n}^{2 / 3}$ must be proportional also to $\mathrm{T}_{\mathrm{SC}}$. The density of the Cooper-pairs is given by $\mathrm{n} \sim \lambda^{-3}$ and therefore $\mathrm{n}^{2 / 3} \sim \lambda^{-2} \sim T_{\mathrm{SC}}$. The distance between the Cooper pairs, $\lambda$, at $\mathrm{T}=\mathrm{T}_{\mathrm{BE}}$, therefore is given by

$$
\lambda \sim \mathrm{T}_{\mathrm{SC}}^{-1 / 2}
$$

In other words, the distance between the Cooper pairs, $\lambda$, is the larger the lower the superconducting transition temperature, $\mathrm{T}_{\mathrm{SC}}$, is. This is a consequence of the weaker coupling of the Cooper-pairs and therefore of their larger size, when $\mathrm{T}_{\mathrm{SC}}$ is low. Using the numerical factors of Eq. (2) and setting $\mathrm{T}_{\mathrm{BE}}=0.135 \cdot \mathrm{T}_{\mathrm{SC}}$ (Figure 9) it follows for $\lambda$ in $\mathrm{nm}$ :

$$
\lambda=104 \cdot \mathrm{T}_{\mathrm{SC}}{ }^{-1 / 2}
$$

This relation, obtained from the observed $\mathrm{T}_{\mathrm{BE}}$ values, replacing in Eq. (2) $\mathrm{T}_{\mathrm{BE}}$ by $\mathrm{T}_{\mathrm{BE}}=0.135 \cdot \mathrm{T}_{\mathrm{SC}}$ (Figure 9), agrees -in fact- nearly quantitatively with direct measurements of the London penetration depths, $\lambda_{\mathrm{L}}$, of the superconducting elements (Figure 10) [17]. Note that the London penetration depth is obtained with a completely different experimental method. The larger pre-factor of 104 compared to 90 in Figure 10 could be due to the fact that the $\lambda$-values in Eq. (4) refer to the finite temperature of $\mathrm{T}_{\mathrm{BE}}$ and not to $\mathrm{T}=0$. However, since the fitted $\mathrm{T}_{\mathrm{BE}}$ values are much lower than $\mathrm{T}_{\mathrm{SC}}$, the Cooper pair density, $\mathrm{n}$, calculated from $\mathrm{T}_{\mathrm{BE}}$ according to Eq. (2) can be considered as the density for $\mathrm{T} \rightarrow 0$. The $\lambda_{\mathrm{L}}$ values in Figure 10 are also representative for $\mathrm{T} \rightarrow 0$ [17]. Additionally, the assumption that the Cooper pairs are closely packed might not be completely correct.

Assuming, nevertheless, that for a dense gas of Cooperpairs the distance between the Cooper-pairs equals their diameter, we can identify the London penetration depth $\lambda_{\mathrm{L}}$ with the diameter of the Cooper-pairs, $\lambda$. This result provides a reasonable interpretation of the London penetration depth, $\lambda_{\mathrm{L}}$.

It has to be noted that the London theory [36,37] was developed in 1935, i.e. much before the postulation of the Cooper pairs in 1957 [1] and their consecutive experimental verification $[3,4,17]$. As a consequence, the London theory treats on unpaired conduction electrons assuming a free electron gas. Using the expression for the London penetration depth $[36,37]$ :

$$
\lambda_{\mathrm{L}}=\left[\mathrm{m}_{\mathrm{e}} /\left(\mu_{0} \cdot \mathrm{q}_{\mathrm{e}}^{2} \cdot \mathrm{n}_{\mathrm{e}}\right)\right]^{1 / 2}
$$

a single-electron density of $\mathrm{n}_{\mathrm{e}}=9.7 \cdot 10^{21} \mathrm{~cm}^{-3}$ is obtained inserting $\lambda_{\mathrm{L}}=54 \mathrm{~nm}$, as calculated for a typical BE temperature of $\mathrm{T}_{\mathrm{BE}}=0.5 \mathrm{~K}$ using Eq. (2). Note that according to Figure $9, \mathrm{~T}_{\mathrm{BE}}=0.5 \mathrm{~K}$ conforms to a superconductor with $\mathrm{T}_{\mathrm{SC}} \sim 3.7 \mathrm{~K}$.

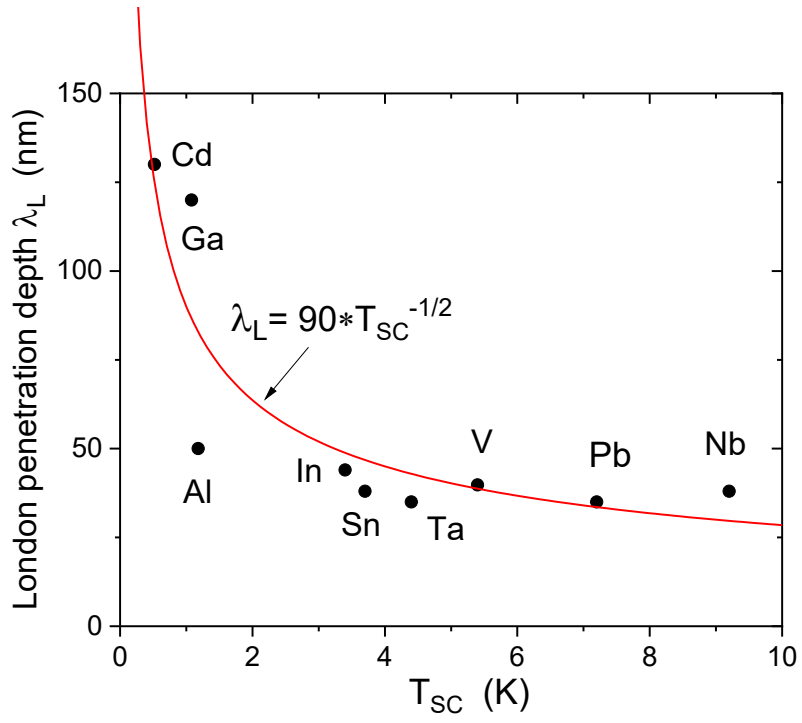

Figure 10. Experimental London penetration depths, $\lambda_{L}$, of the superconducting elements at $T \sim 0 \mathrm{~K}$ as a function of the superconducting transition temperature $T_{S C}$ [17]. These data can reasonably be described by $\lambda_{L}=90 \cdot T_{S C}{ }^{-1 / 2}$ with $\lambda_{L}$ in nanometer.

The hypothetical single-electron density of the London theory, $\mathrm{n}_{\mathrm{e}}$, needed to shield a magnetic field is larger by about a factor of $10^{6}$ compared to the density of the Cooper-pairs of $\mathrm{n}=6.4 \cdot 10^{15} \mathrm{~cm}^{-3}$ calculated using Eq. (2) with $\mathrm{T}_{\mathrm{BE}}=0.5 \mathrm{~K}$. 
As a consequence, in order to explain the strong diamagnetism emerging at $\mathrm{T}_{\mathrm{SC}}$, the London theory has to assume a dramatic increase of the single electron density, $\mathrm{n}_{\mathrm{e}}$, upon entering the superconducting phase. There is no evidence for this dramatic change. The reason for the large difference between the single electron density, $n_{e}$, of the London theory and the Cooper-pair density, n, is the large diameter of the Cooper-pair orbitals and the associated large diamagnetism. For a wave-function of the Cooper-pairs of the isotropic s-state there is no orbital magnetism. Additionally, there is no paramagnetism due to the antiparallel orientation of the spin moments of the two Cooper pair electrons. The field induced orientation of the large diamagnetic orbitals compensates the externally applied magnetic field much more effectively than the unpaired conduction electrons of the assumed free electron gas of the London theory. The assumption of a dense gas of Cooper-pairs with no significant space between them, irrespective of their size, appears justified for the type I superconductors for which no magnetic field lines (vortices) penetrate the sample (Meissner-Ochsenfeld effect). As a result, from the identity $\lambda=\lambda_{\mathrm{L}}$ it follows that only one layer of Cooper pairs next to the inner surface of the sample is sufficient to shield the applied magnetic field completely. We can assume that the electrons of the Cooper-pairs are the only electrons to be considered in the superconducting ground-state, at least for the here considered case of $\mathrm{T} \rightarrow 0$.

The temperature dependence of the superconducting heat capacity has been shown to be boson controlled, as the dynamics of ordered magnets and the lattice dynamics of the non-magnetic solids [19,22]. It is, however, not easy to decide to which extend the superconducting heat capacity is determine by the bosons of the elastic continuum (Debyebosons) or by the bosons of the metallic continuum (the CMS-bosons) because both boson types interact significantly. The observed universal exponents therefore result from a varying proportion of both systems.

Concluding, all nine exponents identified in the superconducting heat capacity should be compiled. Writing these exponents as multiples of $1 / 2$ they are: $3 / 2,4 / 2,5 / 2$, $6 / 2,7 / 2,8 / 2,10 / 2,12 / 2,16 / 2$. As we have mentioned, a quantitative understanding of these exponents is rather difficult.

\section{Conclusions}

From the here observed Bose-Einstein condensation of the Cooper-pairs, the following scenario results: The Cooperpairs are tightly bound objects with a well-defined orbital cross section. The large orbital area is the origin of the strong diamagnetism of the superconductors. However, in contrast to the BE condensation observed for the dilute alkali atom gases, the density of the Cooper-pairs is not a constant but increases with decreasing temperature. The Cooper-pair density calculated from $\mathrm{T}_{\mathrm{BE}}$, therefore, applies to $\mathrm{T}=\mathrm{T}_{\mathrm{BE}}$ but can be taken as representative for $\mathrm{T} \rightarrow 0$. On the one hand, the interaction between the Cooper-pair electrons increases with decreasing temperature, in conformity with the gap energy. The area of the Cooper-pair orbital therefore shrinks. But, on the other hand, the number Cooper pairs seems to increase correspondingly such that the Cooper pairs always form a dense gas of particles. This is condition that no magnetic field lines (vortices) can penetrate the interior of the type I superconductors. Identifying the diameter of the Cooper pair orbital with the London penetration depth it follows that only one layer of Cooper-pairs next to the inner surface of the sample is sufficient to shield an external magnetic field completely. The decreasing penetration depth with decreasing temperature conforms to the decreasing diameter of the Cooper-pair orbital.

\section{Acknowledgments:}

I extend my thanks in deep respect to all of the ingenious experimentalists who have produced the high-precision data used for the analyses of this work.

\section{References:}

[1] J. Bardeen, L.N. Cooper, J.R. Schrieffer, Theory of Superconductivity. Phys. Rev. 108, 1175-1204, 1957.

[2] M. Lang, N. Toyota, T. Sasaki, H. Sato, Magnetic Penetration Depth of $\kappa-(\mathrm{BEDT}-\mathrm{TTF})_{2} \mathrm{Cu}(\mathrm{NCS})_{2}$ : Strong Evidence of Conventional Cooper Pairing, Phys. Rev. Lett. 69, 1443-1446, 1992.

[3] A.M. Kadin, Spatial Structure of the Cooper Pair, J. Supercond. Novel Magnetism, 20, 285-292, 2007.

[4] M. de Llano, F.J. Sevilla S. Tapai, Cooper Pairs as Bosons, Int. J. Mod. Phys. B 20, 2931-2939, 2006.

[5] T. Mishonov, E. Penev, Bernoulli Potential, Hall Constant and Cooper Pairs effective Mass in Disordered BCS Superconductors, Int. J. Mod. Phys. B 17, 28832895, 2003.

[6] S.K. Adhikari, M. Casas, A. Puente, A. Rigo, M. Fortes, M.A. Solis, M. de Llano, A.A. Valladares, O. Rojo, Cooper pair dispersion relation for weak to strong coupling, Phys. Rev. B 62, 8671-8674, 2000.

[7] M. Keller, W. Metzner, U. Schollwöck, Thermodynamics of a superconductor with strongly bound Cooper pairs, Phys. Rev. B 60, 3499-3507, 1999.

[8] A. Tokumitu, K. Miyake, K. Yamada, Cooper-pair and Bose-Einstein condensations in two dimensions: A critical analysis based on the Nozières and SchmittRink formalism, Phys. Rev. B 47, 11988-12003, 1993.

[9] Ze Cheng, Bose-Einstein Condensation of Nonideal Cooper Pairs in the Hartree-Fock-Popov Theory, Found. Phys. 46, 915-942 2016.

[10] K.G. Wilson, J. Kogut, The Renormalization Group Theory and the $\varepsilon$ Expansion. Phys. Rep. 12C, 75-199, 1974.

[11] A. Hoser, U. Köbler, Boson Fields in Ordered Magnets, Acta Phys. Pol. A 127, 350-352, 2015.

[12] U. Köbler, Bosonic and magnonic magnon dispersions, J. Magn. Magn. Mater. 502, 166533, 2020.

[13] U. Köbler, On the Distinction between Debye Bosons and Acoustic Phonons. Int. J. of Thermo. 18, 277284, 2015.

[14] U. Köbler, On the Thermal Conductivity of Metals and of Insulators. Int. J. of Thermo. 20, 210-218, 2017. 
[15] U. Köbler, A. Hoser, Experimental Studies of Boson Fields in Solids, Singapore: World Scientific, 2018.

[16] J.L. Luo et al., Low-Temperature Specific Heat of Superconducting $\mathrm{MgB}_{2}$. Chin. Phys. Lett. 18, 820-822, 2001

[17] R. Kleiner, W. Buckel, Superconductivity: an introduction, Weinheim: Wiley-VCH, 2016.

[18] W.S. Corak, M.P. Garfunkel, C.B. Satterthwaite, A. Wexler, Atomic Heats of Copper Silver and Gold from $1{ }^{0} \mathrm{~K}$ to $5{ }^{0} \mathrm{~K}$. Phys. Rev. 98, 1699-1708, 1955.

[19] U. Köbler, The Importance of the Debye Bosons (Sound Waves) for the Lattice Dynamics of Solids. Int. J. of Thermo. 23, 59-79, 2020.

[20] B.B. Goodman, E. Mendoza, The critical magnetic fields of aluminum, cadmium, gallium and zinc. The London, Edinburgh and Dublin Philosophical Magazine, 42, 594-602, 1951.

[21] U. Köbler, Crossover phenomena in the critical range near magnetic ordering transition, J. Magn. Magn. Mater. 453, 17-29, 2018.

[22] U. Köbler, Universality in the Temperature Dependence of the Heat Capacity of Magnetic Solids. Int. J. of Thermo. 23, 147-174, 2020.

[23] C.B. Satterthwaite, Thermal Conductivity of Normal and Superconducting Aluminum. Phys. Rev. 125, $873-876,1962$.

[24] B.J.C. van der Hoeven, Jr., P.H. Keesom, Specific Heat of Lead and Lead Alloys. Phys. Rev. 137, A103A107, 1965.

[25] Ch. Enss, S. Hunklinger, Low-Temperature Physics, Berlin: Springer, 2005.

[26] C.J. Pethick, H. Smith, Bose-Einstein Condensation in Dilute Gases, Cambridge: Cambridge University Press, 2008.

[27] D.G. Fried, T.C. Killian, L. Willmann, D. Landhuis, S.C. Moss, D. Kleppner, T.J. Greytak, Bose-Einstein Condensation of Atomic Hydrogen. Phys. Rev. Lett. 81, 3811-3814, 1998.

[28] R. Radebaugh, P.H. Keesom, Low-Temperature Thermodynamic Properties of Vanadium, I. Superconducting and Normal States. Phys. Rev. 149, 209-216, 1966.

[29] N.E. Phillips, Low-Temperature Heat Capacities of Gallium, Cadmium and Copper. Phys. Rev. 134, A385A391, 1964.

[30] N.E. Phillips, Heat Capacity of Aluminum between 0.1 ${ }^{0} \mathrm{~K}$ and $4.0{ }^{0} \mathrm{~K}$. Phys. Rev. 114, 676-686, 1959.

[31] C.A. Bryant, P.H. Keesom, Specific Heat of Indium below $1{ }^{\circ} \mathrm{K}$, Phys. Rev. Lett. 4, 460-462, 1960.
[32] J.R. Clement, E.H. Quinnell, Atomic Heat of Indium below $20^{\circ} \mathrm{K}$, Phys. Rev. 92, 258-267, 1953.

[33] Y.S. Touloukian, R.W. Powell, C.Y. Ho, P.G. Klemens, Thermophysical Properties of Matter, vol. 1: Thermal Conducivity of Metallic Elements and Alloys, New York: IFI/Plenum, 1970.

[34] R.F.S. Hearmon, The elastic constants of crystals and other anisotropic materials. Landolt-Börnstein, vol. III/11, ed. by K.-H. Hellwege and A.M. Hellwege, 1286, Berlin: Springer, 1979.

[35] B.J.C. van der Hoeven, Jr., P.H. Keesom, Specific Heat of Niobium between 0.4 and $4.2^{\circ} \mathrm{K}$, Phys. Rev. 134, A1320-A1321, 1964.

[36] M. v. Laue, F. London, H. London, Zur Theorie der Supraleitung, Z. Physik 96, 359-364, 1935.

[37] F. London, H. London, Supraleitung und Diamagnetismus. Physica 2, 341-354, 1935. 